TO APPEAR IN THE ASTROPHYSICAL JOURNAL, LETTERS

Preprint typeset using LATEX style emulateapj v. 11/10/09

\title{
SUPERSOFT X-RAY PHASE OF SINGLE DEGENERATE TYPE Ia SUPERNOVA PROGENITORS IN EARLY TYPE GALAXIES
}

\author{
IZUMI HACHISU \\ Department of Earth Science and Astronomy, College of Arts and Sciences, University of Tokyo, Komaba 3-8-1, Meguro-ku, Tokyo 153-8902, Japan \\ MARIKO KATO \\ Department of Astronomy, Keio University, Hiyoshi 4-1-1, Kouhoku-ku, Yokohama 223-8521, Japan \\ AND \\ KEN'ICHI NOMOTO \\ Institute for the Physics and Mathematics of the Universe, University of Tokyo, Kashiwanoha 5-1-5, Kashiwa, Chiba 277-8583, Japan \\ to appear in the Astrophysical Journal, Letters
}

\begin{abstract}
In the single degenerate (SD) scenario for Type Ia supernova (SN Ia) progenitors, an accreting white dwarf (WD) is expected to undergo a supersoft X-ray source (SSS) phase. Recently, Gilfanov \& Bogdán (2010, hereafter GB10) claimed that observed X-ray fluxes of early type galaxies would be too low to be consistent with the prediction of the SD scenario based on rather simple assumptions. We present realistic evolutionary models of SD systems and calculate durations of SSS phases. In most cases, accreting WDs spend a large fraction of time in the optically thick wind phase and the recurrent nova phase rather than the SSS phase. Thus the SSS phase lasts only for a few hundred thousand years. This is by a factor of $\sim 10$ shorter than those adopted by GB10 where the SN Ia progenitor WD was assumed to spend most of its life as a SSS. The theoretical X-ray luminosity of the SSS has a large uncertainty because of the uncertain atmospheric model of mass-accreting WDs and absorption of soft X-rays by the companion star's cool wind material. We thus adopt an average of the observed fluxes of existing symbiotic SSSs, i.e., $\sim 0.4 \times 10^{36} \mathrm{erg} \mathrm{s}^{-1}$ for $0.3-0.7 \mathrm{keV}$. Using these SSS duration and soft X-ray luminosity, we show that the observed X-ray flux obtained by GB10 is rather consistent with our estimated flux in early type galaxies based on the SD scenario. This is a strong support for the SD scenario as a main-contributor of SNe Ia in early type galaxies.

Subject headings: binaries: close — galaxies: evolution — stars: winds, outflows — supernovae: general — $\mathrm{X}$-rays: binaries
\end{abstract}

\section{INTRODUCTION}

Type Ia supernovae ( $\mathrm{SNe}$ Ia) play very important roles in astrophysics as a standard candle to measure cosmological distances as well as the production site of a large part of iron group elements. However, the nature of SN Ia progenitors has not been clarified yet (e.g., Hillebrandt \& Niemeyer 2000; Livio 2000; Nomoto et al. 1997, 2000). It has been commonly agreed that the exploding star is a carbon-oxygen $(\mathrm{C}+\mathrm{O})$ white dwarf (WD) and the observed features of SNe Ia are better explained by the Chandrasekhar mass model than the sub-Chandrasekhar mass model. However, there has been no clear observational indication as to how the WD mass gets close enough to the Chandrasekhar mass for carbon ignition $\left(M_{\mathrm{Ia}}=1.38 M_{\odot}\right.$ in Nomoto 1982), i.e., whether the WD accretes $\mathrm{H} / \mathrm{He}$-rich matter from its binary companion [single degenerate (SD) scenario] or two $\mathrm{C}+\mathrm{O}$ WDs merge [double degenerate (DD) scenario].

The X-ray signature of these two possible paths are very different. It is believed that no strong X-ray emission is expected from the merger scenario until shortly before the SN Ia explosion. On the other hand, the accreting WD becomes a supersoft X-ray source (SSS) long before the SN Ia explosion, for a million years. In order to constrain progenitor models in early type galaxies, Gilfanov \& Bogdán (2010, here- after, GB10) recently obtained the $0.3-0.7 \mathrm{keV}$ soft X-ray luminosity $L_{\mathrm{X}, \mathrm{obs}}$ and the $K$-band luminosity $L_{K}$ for several early type galaxies. They compared $L_{X, o b s}$ with those predicted from the SN Ia birth rate estimated from $L_{K}$. Their predicted X-ray luminosity $L_{\mathrm{X} \text {,SSS }}$ from the SD scenario is $40-70$ times larger than $L_{\mathrm{X}, \mathrm{obs}}$ and they concluded that no more than five percent of SNe Ia in early type galaxies can be produced by mass-accreting WDs of the SD scenario. However, their $L_{\mathrm{X}, \mathrm{SSS}}$ is based on the following assumptions, which involves large uncertainties. They assumed that (1) all the accreting WDs are in the SSS phase which typically lasts for two million years before a SN Ia explosion and (2) the observed (including absorption) $0.3-0.7 \mathrm{keV}$ soft $\mathrm{X}$-ray flux is as large as $(3-5) \times 10^{36} \mathrm{erg} \mathrm{s}^{-1}$ per source.

In a canonical SD scenario (e.g., Hachisu et al. 1999a), however, the accreting WDs usually spend a large fraction of the lifetime in the optically thick wind phase and in the recurrent nova phase, so that a duration of the SSS phase is much shorter than that assumed by GB10. Moreover, the X-ray luminosity of the symbiotic SSS has a large uncertainty because of the uncertain atmospheric model of mass-accreting WDs and absorption of soft X-rays by the companion star's cool wind material. In these situations, it is reasonable that we use observed fluxes of existing symbiotic SSSs.

In this Letter, we show that the soft X-ray fluxes observed in early type galaxies are consistent with those expected from the SD model, if we adopt a realistic scenario of binary evolutions including a shorter SSS duration and a much more absorbed 


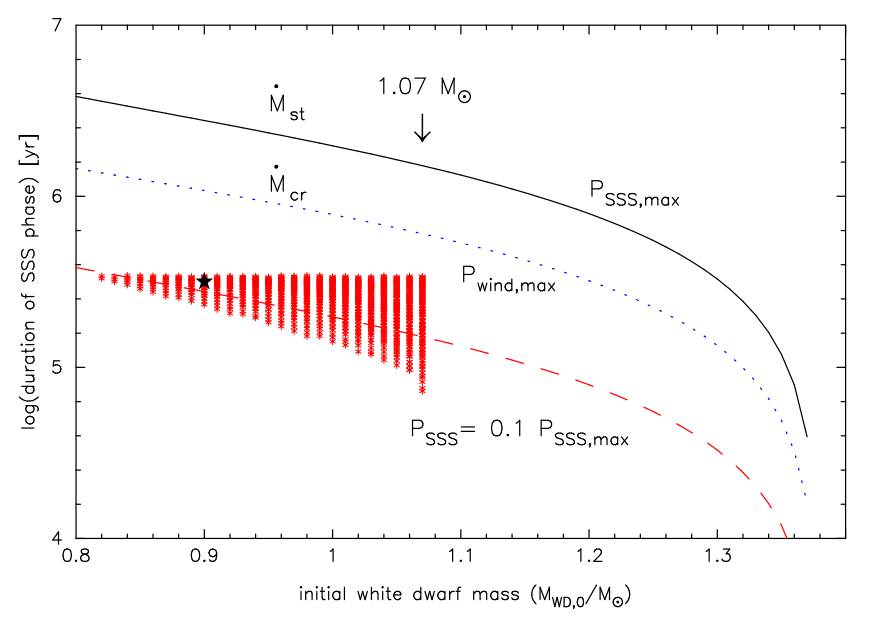

FIG. 1.- Duration of a SSS phase against the initial WD mass for the WD+RG systems. Red asterisks denote the SSS durations of our progenitor models that produced a SN Ia. Solid: $P_{S S S \text { max }}$, the maximum duration of the SSS phase calculated from Equation (4). Dotted: $P_{\text {wind.max }}$, the maximum duration of the wind phase calculated from Equation [5]. Dashed: $P_{\mathrm{SSS}}=$ $0.1 P_{\mathrm{SSS}, \max }$. Star symbol: a model corresponding to Figure 2

soft X-ray flux estimated from existing symbiotic SSSs. We first show the short SSS duration of our SD models in Section 2. In Section 3, we estimate absorbed soft X-ray fluxes in early type galaxies. Discussion follows in Section 4.

\section{DURATION OF SUPERSOFT X-RAY SOURCE PHASE}

In early type galaxies, star formation virtually stopped several Gyr ago. Therefore, only the white dwarf (WD) + red giant (RG) binary systems with the initial RG companion mass of $M_{2,0} \lesssim 1.3 M_{\odot}$ (e.g., Hachisu et al. 1999a) contribute to SNe Ia. For the white dwarf (WD) + main-sequence (MS) star binary systems, more massive companions (usually $M_{2,0} \gtrsim 1.8 M_{\odot}$, see, e.g., Hachisu et al. 1999b) are required but there remain no such massive ones because they have already evolved off. Therefore, here we estimate the duration of a supersoft X-ray phase only for the WD+RG systems.

Our evolutionary model for the SN Ia progenitor is as follows: The binary evolution starts from zero-age mainsequence star pairs with a given set of the primary mass $\left(M_{1, i}\right)$, secondary mass $\left(M_{2, i}\right)$, and separation $\left(a_{i}\right)$ as done in Hachisu et al. (1999a). Unless the initial separation of the binary components is too close, the more massive (primary) component evolves to a RG star (with a helium core) or an AGB star (with a $\mathrm{C}+\mathrm{O}$ core) and fills its Roche lobe or blows a superwind. If subsequent mass transfer from the primary to the secondary is rapid enough to form a common envelope, the binary separation shrinks greatly owing to mass and angular momentum losses from the binary system during the common envelope evolution (see, e.g., Figure 1 of Hachisu et al. 1999a, for an illustration of the binary evolution). The hydrogen-rich envelope of the primary component is stripped away and the primary becomes either a helium star or a $\mathrm{C}+\mathrm{O}$ WD. The helium star further evolves to a $\mathrm{C}+\mathrm{O} \mathrm{WD}$ after a large part of helium is exhausted by core helium burning. Thus we have a binary pair of the $\mathrm{C}+\mathrm{O} \mathrm{WD}$ and the secondary star that is still an MS. At this stage (denoted by 0 ), the binary becomes a pair of the $\mathrm{C}+\mathrm{O}$ WD with the mass of $M_{\mathrm{WD}, 0}$ and the secondary star that is still on the MS; the mass of the secondary star, $M_{2,0}$, is still close to $M_{2,0} \approx M_{2, i}$, because the accreted mass during the common envelope phase is negligibly small.

After the secondary evolves to fill its Roche lobe, the WD accretes mass from the secondary and grows to the critical mass $\left(M_{\mathrm{Ia}}=1.38 M_{\odot}\right.$ in Nomoto 1982) to explode as a SN Ia if the initial binary orbital period $\left(P_{0}\right)$ and the initial mass of the secondary $\left(M_{2,0}\right)$ are in the regions (labeled "initial") shown in Figure 1 of Hachisu et al. (2008). There are two separate regions; one is for binaries consisting of a WD and an MS (WD+MS) and the other is binaries consisting of a WD and a RG (WD+RG). In this figure, the metallicity and the initial mass of the WD were assumed to be $Z=0.02$ and $M_{\mathrm{WD}, 0}=1.0 M_{\odot}$. In early type galaxies considered here, however, MS stars more massive than $1.3 M_{\odot}$ have already evolved off to RGs or WDs, so that only the WD+RG systems can produce $\mathrm{SNe}$ Ia.

After the mass transfer begins from the RG to the WD in our WD+RG systems, a steady-state supersoft X-ray source (SSS) phase can be realized only when the mass accretion rate onto the WD satisfies

$$
\dot{M}_{\mathrm{st}}<\dot{M}_{\mathrm{WD}}<\dot{M}_{\mathrm{cr}},
$$

where $\dot{M}_{\mathrm{st}}$ is the lower limit mass accretion rate for stable hydrogen shell burning on the WD, $\dot{M}_{\mathrm{WD}}$ the mass accretion rate onto the WD, and $\dot{M}_{\mathrm{cr}}$ the critical mass accretion rate above which the WD envelope expands to blow optically thick winds (Hachisu et al. 1996, 1999a b). Nomoto et al. (2007) obtained these critical rates as

$$
\dot{M}_{\text {st }}=3.066 \times 10^{-7}\left(\frac{M_{\mathrm{WD}}}{M_{\odot}}-0.5357\right) M_{\odot} \mathrm{yr}^{-1},
$$

and

$$
\dot{M}_{\mathrm{cr}}=6.682 \times 10^{-7}\left(\frac{M_{\mathrm{WD}}}{M_{\odot}}-0.4453\right) M_{\odot} \mathrm{yr}^{-1} .
$$

Using Equation (1), we estimate the "maximum duration" of the steady-state SSS phase before the SN Ia explosion, i.e.,

$P_{\mathrm{SSS}, \text { max }}=\int_{M_{\mathrm{WD}, 0}}^{M_{\mathrm{Ia}}} \frac{d M}{\dot{M}_{\mathrm{st}}}=3.3 \times 10^{6} \ln \left(\frac{M_{\mathrm{Ia}} / M_{\odot}-0.5357}{M_{\mathrm{WD}, 0} / M_{\odot}-0.5357}\right) \mathrm{yr}$.

We plot the value of $P_{\text {SSS,max }}$ (solid line) in Figure 1 against various initial WD masses. If there are any paths in binary evolutions in which the mass accretion rate would always follow Equation (2), its SSS duration would be given by this $P_{\text {SSS,max }}$. We should note that GB10 assumed essentially the same SSS duration as this $P_{\mathrm{SSS}, \max }$. For comparison, we also plot the maximum duration of the wind phase, $P_{\text {wind,max }}$ (dotted line), i.e.,

$P_{\text {wind, } \max }=\int_{M_{\mathrm{WD}, 0}}^{M_{\mathrm{Ia}}} \frac{d M}{\dot{M}_{\mathrm{cr}}}=1.5 \times 10^{6} \ln \left(\frac{M_{\mathrm{Ia}} / M_{\odot}-0.4453}{M_{\mathrm{WD}, 0} / M_{\odot}-0.4453}\right) \mathrm{yr}$.

The upper limit mass for $\mathrm{C}+\mathrm{O}$ WDs born in binary systems was estimated to be $\sim 1.07 M_{\odot}$ by Umeda et al. (1999). In early type galaxies, the donor star is relatively less massive and cannot supply much mass to the WD. Therefore, the initial mass of WDs should be as massive as $0.8 M_{\odot}$ or more as shown in Figures 12 and 13 of Hachisu et al. (1999a). Here we adopt an upper limit of $M_{\mathrm{WD}, 0}=1.07 M_{\odot}$ for the initial WD mass. We have followed binary evolutions using the same method and physical parameters as those in Hachisu et al. (1999a).

In Figures 2 and 3, a typical example of the evolutionary path is plotted, i.e., $M_{\mathrm{WD}, 0}=0.9 M_{\odot}, M_{2,0}=1.3 M_{\odot}$, and 


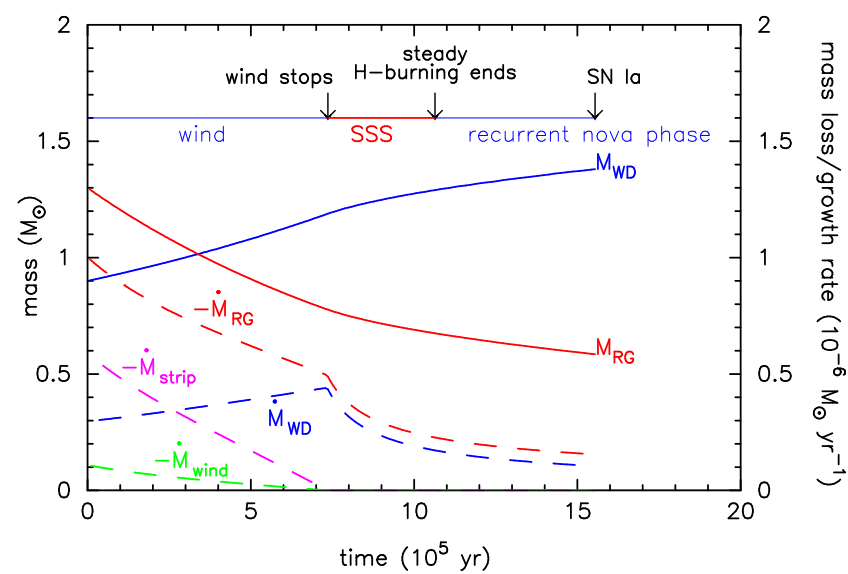

FIG. 2.- Time evolution of a SN Ia progenitor system for an initial set of parameters of $M_{\mathrm{WD}, 0}=0.9 M_{\odot}, M_{2,0}=1.3 M_{\odot}$, and $a_{0}=297 R_{\odot}\left(P_{0}=400\right.$ days). The WD blows optically thick winds until $t=P_{\text {wind }} \sim 7.3 \times 10^{5} \mathrm{yr}$, enters the SSS phase until $\sim 10.5 \times 10^{5} \mathrm{yr}$, then becomes a recurrent nova, and finally explodes as a SN Ia at $t_{\mathrm{SN} \text { Ia }} \sim 15.5 \times 10^{5}$ yr. We obtain the duration of $P_{\mathrm{SSS}} \sim 3.2 \times 10^{5} \mathrm{yr}$. We include the rate of mass-stripping from the RG by the WD wind ( $\dot{M}_{\text {strip }}$, that is, $-\dot{M}_{\mathrm{RG}} \approx-\dot{M}_{\text {wind }}-\dot{M}_{\text {strip }}+\dot{M}_{\mathrm{WD}}$ during the wind phase) and the mass-loss during helium shell-flashes $\left(\dot{M}_{\mathrm{WD}}=\right.$ $\eta_{\mathrm{H}} \eta_{\mathrm{He}}\left|\dot{M}_{\mathrm{RG}}-\dot{M}_{\text {strip }}\right|$ ). See Equations (22) and (15) of Hachisu et al. (1999a), respectively. The WD and RG masses (solid lines) refer to the left axis while the mass-loss/growth rates (dashed lines) refers to the right axis.

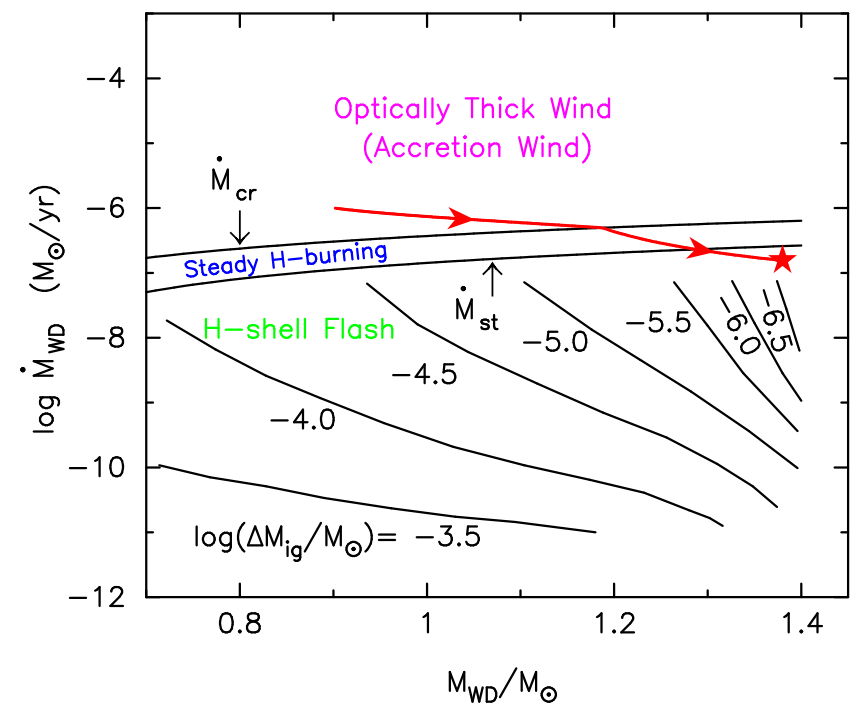

FIG. 3.- Evolutionary path (red solid) of a SN Ia progenitor (same model as in Figure 2] on the map of response of white dwarfs to mass accretion rate. The progenitor explodes at the star mark as a SN Ia. Strong optically thick winds blow above the line of $\dot{M}_{\mathrm{WD}}>\dot{M}_{\mathrm{cr}}$. The wind mass-loss rate is $\dot{M}_{\text {wind }} \approx \dot{M}_{\mathrm{WD}}-\dot{M}_{\mathrm{cr}}$. Steady hydrogen shell burning with no optically thick winds occur between $\dot{M}_{\mathrm{st}} \leq \dot{M}_{\mathrm{WD}} \leq \dot{M}_{\mathrm{cr}}$. There is no steady state burning below $\dot{M}_{\mathrm{WD}}<\dot{M}_{\text {st. }}$. Instead, intermittent shell flashes occur. The envelope mass, $\Delta M_{\mathrm{ig}}$, at which a hydrogen shell flash ignites, is also shown (taken from Figure 9 of Nomoto 1982). Progenitor's crossing time of the region between $\dot{M}_{\mathrm{st}} \leq \dot{M}_{\mathrm{WD}} \leq \dot{M}_{\mathrm{cr}}$, i.e., the duration of a SSS phase, is relatively short.

$P_{0}=400$ days $\left(a_{0}=297 R_{\odot}\right)$. In these figures, the evolution starts when the secondary RG fills its Roche lobe to start mass transfer. The mass transfer rate at the initial phase exceeds $\dot{M}_{\text {cr }}$, so that the WD blows optically thick winds until $t=$ $P_{\text {wind }} \sim 7.3 \times 10^{5} \mathrm{yr}$. Then the WD enters the SSS phase until $t \sim 10.5 \times 10^{5} \mathrm{yr}$, followed by the recurrent nova $(\mathrm{RN})$ phase, and finally explodes as an SN Ia at $t_{\mathrm{SN} \text { Ia }} \sim 15.5 \times 10^{5} \mathrm{yr}$. In the RN phase, the WD undergoes weak hydrogen shell-flashes every 10 yrs or so. We may observe a SSS phase at every RN explosion but this duration is very short, only $0.6 \%$ of the mass accretion phase, for example, in the RS Oph case (50 days against 22 yrs; see, e.g., Hachisu et al. 2007). In this case, we have a duration of $P_{\mathrm{SSS}} \sim 3.2 \times 10^{5} \mathrm{yr}$, i.e., the crossing time of the region between $\dot{M}_{\mathrm{st}} \leq \dot{M}_{\mathrm{WD}} \leq \dot{M}_{\mathrm{cr}}$ is $\sim 10$ times shorter than that estimated from Equation (4).

We have also examined $P_{\text {SSS }}$ for 1239 cases with a different set of initial parameters as shown by red asterisks in Figure 1 The lower boundary $\left(P_{\mathrm{SSS}} \sim 0.7-3.3 \times 10^{5} \mathrm{yr}\right)$ is mainly determined by the upper limit of the companion mass, i.e., $1.3 M_{\odot}$, while the upper boundary $\left(P_{\mathrm{SSS}} \sim 3.4 \times 10^{5} \mathrm{yr}\right)$ is determined by the lowest companion mass that depends on the orbital period as shown in Figures 12 and 13 of Hachisu et al. (1999a). The mean duration of these cases, which covers our progenitor regions of SNe Ia, is $P_{\mathrm{SSS}} \sim 2.5_{-1.8}^{+0.9} \times 10^{5} \mathrm{yr}$. Therefore, we adopt a typical value of $P_{\text {SSS }} \sim 2.5 \times 10^{5} \mathrm{yr}$ in our estimate, which is a factor of $\sim 8$ shorter than the value assumed by GB10.

\section{SOFT X-RAY FLUX IN THE SD MODEL}

GB10 obtained the $0.3-0.7 \mathrm{keV}$ soft X-ray to $K$-band luminosity ratio for several early type galaxies and compared them with the X-ray luminosity expected from the SN Ia birth rate. They assumed the number of accreting WDs in the SSS phase as

$$
N_{\mathrm{WD}, \mathrm{SSS}} \approx \frac{\Delta M_{\mathrm{WD}}}{\dot{M}_{\mathrm{WD}}} \dot{N}_{\mathrm{SN} \text { Ia }} \approx P_{\mathrm{SSS}} \dot{N}_{\mathrm{SN} \text { Ia }},
$$

where $\Delta M_{\mathrm{WD}}$ is the mass difference between the initial mass $\left(M_{\mathrm{WD}, 0}\right)$ and the final mass $\left(M_{\mathrm{Ia}}\right)$, and $\dot{N}_{\mathrm{SN}}$ Ia is the SN Ia birth rate given by

$$
\dot{N}_{\mathrm{SN} \text { Ia }}=\frac{1}{2} \times 3.5 \times 10^{-4}\left(\frac{L_{K}}{10^{10} L_{K, \odot}}\right) \mathrm{yr}^{-1} .
$$

If we adopt our real duration of $P_{\mathrm{SSS}} \sim 2.5 \times 10^{5} \mathrm{yr}$, the number of WDs in the SSS phase is

$$
N_{\mathrm{WD}, \mathrm{SSS}} \approx 44\left(\frac{L_{K}}{10^{10} L_{K, \odot}}\right),
$$

as tabulated in Table 1 together with the results of GB10. Since our real duration of $P_{\mathrm{SSS}}$ is much shorter than those assumed by GB10, the number of accreting WDs is by a factor of $\sim 7$ smaller than those obtained by GB10.

We also point out that the soft X-ray flux from massaccreting WDs in the SSS phase involves quite a large uncertainty. The key point is that the progenitor system is the WD+RG system, in which the WD is embedded in a complex circumbinary matter. Furthermore, we have not yet fully understood atmospheric structures of mass-accreting WDs in symbiotic stars (e.g., Jordan et al. 1996; Orio et al. 2007). In these situations, it is reasonable that we use observed fluxes of symbiotic SSSs with known distance like the member of the Small Magellanic Cloud (SMC). There are two well-studied symbiotic SSSs in the SMC, SMC 3 and Lin 358.

The soft X-ray flux $(0.15-2.4 \mathrm{keV})$ from SMC 3 with ROSAT was obtained to be $\sim 3.3 \times 10^{36}(d / 65 \mathrm{kpc})^{2} \mathrm{erg} \mathrm{s}^{-1}$ by Kahabka et al. (1994). The flux between $0.3-0.7 \mathrm{keV}$ is $0.3-0.4$ of the total flux (see, e.g., Figures 2-6 of Jordan et al. 1996, for the spectrum), so that $\ell_{\mathrm{X}, \mathrm{obs}} \sim 1 \times 10^{36} \mathrm{erg} \mathrm{s}^{-1}$ for 
$0.3-0.7 \mathrm{keV}$. This Kahabka et al.'s flux has been already corrected for the Galactic absorption, i.e., $N_{\mathrm{H}}=3 \times 10^{20} \mathrm{~cm}^{-2}$ with an unknown factor, which is not specified in the paper. Thus the absorbed flux at the Earth is smaller than $1 \times 10^{36} \mathrm{erg} \mathrm{s}^{-1}$. The $0.2-1.0 \mathrm{keV}$ flux from SMC 3 outside eclipse was also observed with XMM-Newton, and Orio et al. (2007) obtained $\sim 3 \times 10^{-12} \mathrm{erg} \mathrm{cm}^{-2} \mathrm{~s}^{-1}$ at the Earth (private communication). The $0.3-0.7 \mathrm{keV}$ flux is about $60 \%$ of the total flux (see Figure 2 of Orio et al. 2007, for the spectrum), i.e., $f_{\mathrm{X}, \mathrm{obs}}=1.8 \times 10^{-12} \mathrm{erg} \mathrm{cm}^{-2} \mathrm{~s}^{-1}$. We obtain $\ell_{\mathrm{X}, \mathrm{obs}}=$ $4 \pi d^{2} f_{\mathrm{X}, \mathrm{obs}}=7.7 \times 10^{35}(d / 60 \mathrm{kpc})^{2} \mathrm{erg} \mathrm{s}^{-1}$. The above Kahabka et al.'s value is consistent with this flux, suggesting no variation of the X-ray flux during $\sim 10 \mathrm{yr}$. So, in the following, we use Orio et al.'s flux of $\ell_{\mathrm{X}, \mathrm{obs}}=7.7 \times 10^{35} \mathrm{erg} \mathrm{s}^{-1}$. Adopting neutral hydrogen $N_{\mathrm{H}}=4.9 \times 10^{20} \mathrm{~cm}^{-2}$ and temperature $T=5.1 \times 10^{5} \mathrm{~K}$ (Rauch model for MOS- 1 data in Table 1 in Orio et al. 2007), we have calculated the correction factor of the absorbed flux with different $N_{\mathrm{H}}$ but the same temperature. For example, the correction factor for NGC3585, $N_{\mathrm{H}}=5.6 \times 10^{20} \mathrm{~cm}^{-2}$ (Bogdán \& Gilfanov 2010), is calculated to be 0.88 against LMC 3 of $7.7 \times 10^{35} \mathrm{erg} \mathrm{s}^{-1}$ assuming blackbody of $T=5.1 \times 10^{5} \mathrm{~K}$, so we have the expected absorbed flux from NGC3585 to be $\ell_{\mathrm{X}, \mathrm{cal}}=6.8 \times 10^{35} \mathrm{erg} \mathrm{s}^{-1}$. In this way, we have obtained expected absorbed flux for each early type galaxy listed in Table 1

Another example is Lin 358, the Small Magellanic Cloud symbiotic star. The absorbed soft X-ray flux $(0.13-1.0 \mathrm{keV})$ from Lin 358 with XMM Newton was estimated to be $8.3 \times 10^{34}(d / 60 \mathrm{kpc})^{2} \mathrm{erg} \mathrm{s}^{-1}$ with $N_{\mathrm{H}}=7.6 \times 10^{20} \mathrm{~cm}^{-2}$ and $T=2.3 \times 10^{5} \mathrm{~K}$ by Kahabka \& Haberl (2006). Since the spectrum is very soft, the flux between $0.3-0.7 \mathrm{keV}$ is at most 0.2 of the total flux (see Figure 3 of Orio et al. 2007, for the spectrum). We obtain $\ell_{\mathrm{X}, \mathrm{obs}} \sim 1.7 \times 10^{34} \mathrm{erg} \mathrm{s}^{-1}$ for $0.3-0.7$ $\mathrm{keV}$. The converted factor to NGC3585 is 1.6 and we obtain the expected X-ray flux to be $\ell_{\mathrm{X}, \mathrm{cal}}=2.7 \times 10^{34} \mathrm{erg} \mathrm{s}^{-1}$.

These two symbiotic stars have very different values of $X$ ray flux, so we adopt an arithmetic mean of these two and each $\ell_{\mathrm{X}, \mathrm{cal}}$ is shown in Table 1 Then the total expected supersoft $\mathrm{X}$-ray luminosity of $L_{\mathrm{X}, \mathrm{sSS}}$ is obtained as

$$
L_{\mathrm{X}, \mathrm{SSS}}=N_{\mathrm{WD}, \mathrm{SsS}} \times \ell_{\mathrm{X}, \mathrm{cal}},
$$

for the expected soft $(0.3-0.7 \mathrm{keV}) \mathrm{X}$-ray flux, that is also shown in Table 1. The estimated soft X-ray fluxes are rather consistent with the observed ones of early type galaxies. This is in apparent contradiction with the claim made by GB10.

\section{DISCUSSION}

Our estimated duration of $P_{\text {SSS }} \sim 2.5_{-1.8}^{+0.9} \times 10^{5} \mathrm{yr}$ is rather stiff as long as our SD model is concerned. However, there is still a large uncertainty on the absorbed soft X-ray flux. The bright symbiotic SSSs are rather rare as Vogel \& Morgan (1994) wrote "Its (SMC 3) X-ray luminosity in the 0.1-2.4 $\mathrm{keV}$ energy band was estimated to be $\approx 400 L_{\odot}$. This is an unusually high X-ray flux for a symbiotic binary.” If SMC 3 is a brightest exception considering low X-ray fluxes of other symbiotic SSSs like Lin 358, our flux estimate in Table1 1 may be reduced further.

As one of new possible mechanisms to SNe Ia, which might be additional soft X-ray fluxes, King et al. (2003) speculated that a dwarf nova could become a SSS during the outburst if $\dot{M}_{\mathrm{WD}}$ temporarily increases by a factor of $\sim 10-100$ (i.e., $\dot{M}_{\text {WD }}>\dot{M}_{\text {st }}$ ). Such a SSS, however, cannot be realized. Hydrogen shell-burning does not occur in such a case because the accreted envelope mass is too small to be ignited. For example, Starrfield et al. (1988) calculated mass accretion onto the very massive $\mathrm{WD}$ of $1.35 M_{\odot}$. With a high accretion rate of $1.1 \times 10^{-6} M_{\odot} \mathrm{yr}^{-1}$, they found that hydrogen ignites $2.6 \mathrm{yr}$ after the accretion starts. This means that the accreted mass of $3 \times 10^{-6} M_{\odot}$ should be required before the hydrogen ignition.

In King et al.'s idea (see their Figure 2), the average mass accretion rate is $1 \times 10^{-9} M_{\odot} \mathrm{yr}^{-1}$ and the duty cycle is 0.004 , which means a burst mass accretion rate of $2.5 \times 10^{-7} M_{\odot} \mathrm{yr}^{-1}$ $\left(>\dot{M}_{\mathrm{st}}\right)$. The viscous timescale of the accretion disk in the WD+RG systems like RS Oph is about a few to several hundred days or so (e.g., King \& Pringle 2009). Therefore, the disk mass can be estimated to be $2.5 \times 10^{-7} M_{\odot} \mathrm{yr}^{-1} \times(0.7-$ 1.5) $\mathrm{yr} \sim(2-4) \times 10^{-7} M_{\odot}$, which is too small to trigger a shell burning even on a very massive WD like in RS Oph. Even if a disk instability occurs, the accreted matter simply accumulates on the WD but remains unburnt. After a few tens of disk instabilities, the accreted materials finally reaches the critical mass of $3 \times 10^{-6} M_{\odot}$ and results in a shell flash. This is nothing else a classical (or recurrent) nova and its SSS duration is too short to be compared with the steady SSS duration as mentioned earlier.

We thank Marat Gilfanov and Joanna Mikołajewska for useful discussion on symbiotic SSSs like SMC 3. We are also grateful to the anonymous referees for their detailed comments that helped to improve the paper. This research has been supported by the Grant-in-Aid for Scientific Research of the Japan Society for the Promotion of Science (20540226, 20540227), and by World Premier International Research Center Initiative, MEXT, Japan.

\section{REFERENCES}

Bogdán, Á., \& Gilfanov, M. 2010, A\&A, 512, 16

Gilfanov, M., \& Bogdán, A. 2010, Nature, 463, 924 (GB10)

Hachisu, I., Kato, M., \& Luna, G. J. M. 2007, ApJ, 659, L153

Hachisu, I., Kato, M., \& Nomoto, K. 1996, ApJ, 470, L97

Hachisu, I., Kato, M., \& Nomoto, K. 1999a, ApJ, 522, 487

Hachisu, I., Kato, M., \& Nomoto, K. 2008, ApJ, 683, L127

Hachisu, I., Kato, M., Nomoto, K., \& Umeda, H. 1999b, ApJ, 519, 314

Hillebrandt, W., \& Niemeyer, J. 2000, ARA\&A, 38, 191

Jordan, S., Schmutz, W., Wolff, B., Werner, K., \& Muerset, U. 1996, A\&A, 312, 897

Kahabka, P., \& Haberl, F. 2006, A\&A, 452, 431

Kahabka, P., Pietsch, W., \& Hasinger, G. 1994, A\&A, 288, 538

King, A. R., Rolfe, D. J., \& Schenker, K. 2003, MNRAS, 341, L35

King, A. R., \& Pringle, J. E. 2009, MNRAS, 397, L51

Livio, M. 2000, Type Ia Supernovae: Theory and Cosmology, (Cambridge: Cambridge Univ. Press), 33
Nomoto, K. 1982, ApJ, 253, 798

Nomoto, K., Iwamoto, K., \& Kishimoto, N. 1997, Science, 276, 1378

Nomoto, K., Saio, H., Kato, M., \& Hachisu, I. 2007, ApJ, 663, 1269

Nomoto, K., Umeda, H., Kobayashi, C., Hachisu, I., Kato, M., \& Tsujimoto, T. 2000, in AIP Conf. Proc. Vol. 522: Cosmic Explosions: Tenth Astrophysics Conference, ed. S. S. Holt \& W. W. Zhang (American Institute of Physics), 35

Orio, M., Zezas, A., Munari, U., Siviero, A., \& Tepedelenlioglu, E. 2007, ApJ, 661, 1105

Starrfield, S., Sparks, W. M., \& Shaviv, G. 1988, ApJ, 325, L35

Umeda, H., Nomoto, K., Yamaoka, H., \& Wanajo, S. 1999, ApJ, 513, 861

Vogel, M., \& Morgan, D. H. 1994, A\&A, 288, 842 
TABLE 1

WHITE DWARFS IN SUPERSOFT X-RAY SOURCE PHASE

\begin{tabular}{|c|c|c|c|c|c|c|}
\hline galaxy & $\begin{array}{c}L_{K}^{\mathrm{a}} \\
\left(10^{10} L_{K, \odot}\right)\end{array}$ & $N_{\mathrm{WD}, \mathrm{SSS}}{ }^{\mathrm{a}}$ & $N_{\mathrm{WD}, \mathrm{SSS}}{ }^{\mathrm{b}}$ & $\begin{array}{c}\ell_{\mathrm{X}, \mathrm{cal}^{\mathrm{c}}} \\
\left(10^{35} \mathrm{erg} \mathrm{s}^{-1}\right)\end{array}$ & $\begin{array}{c}L_{X, \mathrm{obs}^{\mathrm{a}}} \\
\left(10^{37} \mathrm{erg} \mathrm{s}^{-1}\right)\end{array}$ & $\begin{array}{c}L_{\mathrm{X}, \mathrm{Sss}}{ }^{\mathrm{d}} \\
\left(10^{37} \mathrm{erg} \mathrm{s}^{-1}\right)\end{array}$ \\
\hline M32 & 0.085 & 25 & 3.7 & 3.1 & 0.15 & 0.12 \\
\hline NGC 3377 & 2.0 & 580 & 88 & 5.8 & 4.7 & 5.1 \\
\hline M31 bulge & 3.7 & 1100 & 160 & 2.9 & 6.3 & 4.7 \\
\hline M105 & 4.1 & 1200 & 180 & 5.9 & 8.3 & 11 \\
\hline NGC 4278 & 5.5 & 1600 & 240 & 7.2 & 15 & 17 \\
\hline NGC 3585 & 15 & 4400 & 660 & 3.5 & 38 & 23 \\
\hline
\end{tabular}

a Table 1 of GB10

$\mathrm{b}$ this work, Equation 8

$c$ this work

$\mathrm{d}$ this work, Equation 9 